\title{
Simulation of bimolecular reactions: numerical challenges with the graph Laplacian
}

\author{
S. MacNamara ${ }^{1} \quad$ Sergio Blanes ${ }^{2} \quad$ Arieh Iserles $^{3}$
}

(Received 26 February 2020; revised 19 April 2020)

\begin{abstract}
An important framework for modelling and simulation of chemical reactions is a Markov process sometimes known as a master equation. Explicit solutions of master equations are rare; in general the explicit solution of the governing master equation for a bimolecular reaction remains an open question. We show that a solution is possible in special cases. One method of solution is diagonalization. The crucial class of matrices that describe this family of models are non-symmetric graph Laplacians. We illustrate how standard numerical algorithms for finding eigenvalues fail for the non-symmetric graph Laplacians that arise in master equations for models of chemical kinetics. We propose a novel way to explore the pseudospectra of the non-symmetric graph Laplacians that arise in this class of applications, and illustrate our proposal by Monte Carlo. Finally, we apply the Magnus expansion,
\end{abstract}

DOI:10.21914/anziamj.v61i0.15169, (C) Austral. Mathematical Soc. 2020. Published 2020-06-16, as part of the Proceedings of the 14th Biennial Engineering Mathematics and Applications Conference. ISSN 1445-8810. (Print two pages per sheet of paper.) Copies of this article must not be made otherwise available on the internet; instead link directly to the DOI for this article. 
which provides a method of simulation when rates change in time. Again the graph Laplacian structure presents some unique issues: standard numerical methods of more than second-order fail to preserve positivity. We therefore propose a method that achieves fourth-order accuracy, and maintain positivity.

\section{Contents}

1 Introduction

C60

2 Exact solutions, spectra and pseudospectra

C62

3 Application of the Magnus expansion

C67

4 Conclusions

C70

\section{Introduction}

This article is motivated by the experiments in a Harvard chemistry lab with a so-called Dimple Machine, described in "Mass action at the single-molecule level," by Shon and Cohen [12], and on the website http://cohenweb.rc. fas.harvard.edu/Research/TrapSingMol/DimpleMachine.htm. Sections 1.2 and 1.6 of Shon's [11] associated PhD thesis describe the way that Markov processes provide a mathematical framework for models of these chemical reactions, and we use the same mathematical models here. Briefly, we study what is sometimes termed a master equation

$$
\frac{d}{d t} p=\mathbb{A} p \quad \text { with solution } \quad p(t)=\exp (\mathbb{A} t) p(0)
$$

Here the ith component of the vector $p \in \mathbb{R}^{n}$ records the probability of being in state $i$. A state is defined by the integer number of molecules of each type of chemical species. The matrix $\mathbb{A}=\left[a_{i j}\right]$ must be an example of a 
(typically non-symmetric) graph Laplacian, which is defined by the properties that off-diagonal entries are zero or positive, and each column sums to zero:

- $a_{i j} \geqslant 0$ for $i \neq j$; and

- $a_{j j}=-\sum_{i \neq j} a_{i j}$.

Shon and Cohen [12, Figure 4] compared the mathematical model (1) with experiments - these chemical reactions are described extremely well by this theory!

In very special cases a governing master equation has an exact solution, in which case the matrix exponential appearing in (1) can be made more explicit. For example, for a model of monomolecular reactions, exact solutions and eigenvalues of an associated matrix were found by Iserles and MacNamara [5]. This monomolecular model is an example of a class of master equations in which a family of binomial distributions form a one-dimensional invariant manifold, and it has important applications in ion channel kinetics [3].

For the rest of this article we concentrate on the bimolecular reaction

$$
\mathrm{S}_{1}+\mathrm{S}_{2} \leftrightarrows \mathrm{S}_{3}
$$

We assume the initial state is $\left(m_{1}, m_{2}, 0\right)$ molecules of each of the three species, $S_{1}, S_{2}, S_{3}$, with $m_{2} \geqslant m_{1}$. With $n=m_{1}+1$, this reaction is modelled by the $n \times n$ tridiagonal matrix

$$
\mathbb{A}_{i, j}= \begin{cases}c_{2}(j-1), & i=j-1, \\ -c_{2}(j-1)-c_{1}\left(m_{1}-j+1\right)\left(m_{2}-j+1\right), & i=j, \\ c_{1}\left(m_{1}-j+1\right)\left(m_{2}-j+1\right), & i=j+1 .\end{cases}
$$

Here $c_{1}, c_{2}$ denote positive rate constants for the forward and backward directions, respectively, and are denoted $k_{\text {on }}$ and $k_{\text {off }}$ with appropriate units in Table 1 of Shon and Cohen [12]. The exact scalings of the rate constants depend on things such as the volume of the reacting vessel, which will not be considered here, and which do not effect the results we describe, which are 
generally applicable to this class of models. For simplicity, we give examples for the special case $m_{1}=m_{2}=m$, although our methods work more generally. This special case models the experiments in Figure 4(f) of Shon and Cohen [12] which consider $m=1,2,3$ (in their notation, $\left(N_{R}, N_{G}\right)=(1,1),(2,2),(3,3)$ with the number of red monomers $N_{R}=m_{1}$ and green monomers $N_{G}=m_{2}$ ).

\section{Exact solutions, spectra and pseudospectra}

We examine the spectra and pseudospectra of the matrix $\mathbb{A}$ in (2). Then we outline a procedure for exact diagonalization, which leads to more explicit solutions via $\exp (\mathbb{A} t)=V \operatorname{diag}\left(e^{\lambda_{0}}, \ldots, e^{\lambda_{m}}\right) V^{-1}$ (albeit the algebraic expressions for $\mathrm{V}^{-1}$ could quickly become unwieldy). It is helpful to know that, surprisingly, the non-symmetric matrix does not have complex eigenvalues.

Lemma 1. The eigenvalues of the non-symmetric matrix (2) representing the bimolecular reaction and the Dimple Machine are purely real.

Here we use the same method of proof described by Trefethen and Embree [14, Sec. 12], specialised to this particular application of the Dimple Machine.

Proof: Let $d_{1}=1$. Recursively define

$$
d_{i+1}=\left(\frac{c_{2}(j-1)}{c_{1}\left(m_{1}-j+1\right)\left(m_{2}-j+1\right)}\right)^{1 / 2} d_{i} .
$$

Define the diagonal matrix $\mathbb{D}$ with diagonal $d_{i i}=d_{i}$. Direct matrix multiplication confirms that $\mathbb{D} \mathbb{A D}^{-1}$ is a symmetric matrix, so by the Spectral Theorem, eigenvalues are real. Hence the eigenvalues of $\mathbb{A}$ are also real, because $\mathbb{A}$ is similar to $\mathbb{D} \mathbb{A D}^{-1}$.

Remark 2. A numerical calculation of eigenvalues of matrix (2), say for $m>100$, almost always produces complex numbers (in all numerical software, 
including MATLAB), with imaginary components of significant magnitude. See Figure 1. Lemma 1 shows such numerical calculations are wrong. This numerical issue is related to Twisted Toeplitz matrices and the pseudospectra [14]. Pseudospectra and other computational issues for this class of problems are discussed by MacNamara [6], MacNamara, Burrage, and Sidje [9], MacNamara, McLean, and Burrage [10], and MacNamara et al. [7], and references therein.

Figure 1 also shows numerical estimates of eigenvalues for a perturbed version of the matrix (2). These perturbed points are a very crude estimate of the pseudospectra of the matrix, visualised as a dot cloud. However, there is an important difference between what is computed in Figure 1 and the standard definition of the pseudospectra in the literature. For $\epsilon>0$, one equivalent definition of the $\epsilon$-pseudospectra of $A$ is the set (in the complex plane) of eigenvalues of some perturbed matrix $A+E$ :

$$
\left\{\lambda \in \mathbb{C}:(A+E) v=\lambda \nu, \quad E \in \mathbb{C}^{n \times n}, \quad\|E\|<\epsilon\right\} .
$$

The formal notion of the pseudospectra has a drawback for applications that involve graph Laplacian (such as the applications to chemical kinetics via master equations and Markov processes that we describe) because it corresponds to perturbing entries in the matrix $\mathbb{A}$ in a way that does not have a physical interpretation in the probabilistic model. In particular, the above perturbation permits:

- negative off-diagonal entries;

- columns with non-zero sum; and

- off-diagonal entries, that were originally zero, becoming non-zero.

The first two points violate the graph Laplacian structure, and the third point is not allowed according the chemical reactions being modelled by the matrix. For example, changing entries beyond the tridiagonal structure would correspond to changes in state that are impossible for a bimolecular chemical reaction. 


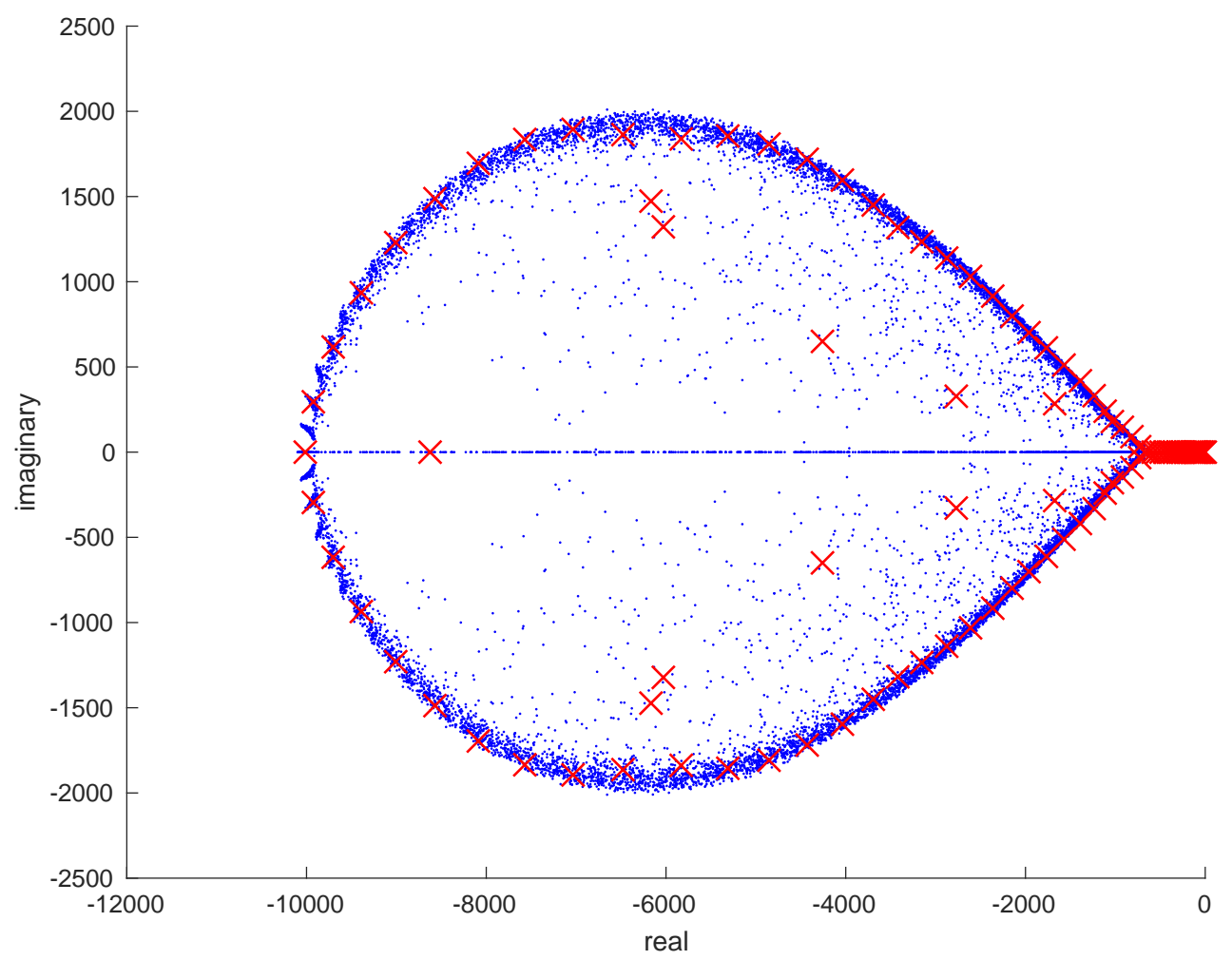

Figure 1: Numerical estimates of the eigenvalues of a $100 \times 100$ instance of the matrix (2) with $c_{1}=c_{2}=1, m=99$, computed with the numerical software MATLAB, plotted (red ' $X$ ') in the complex plane. Near the negative real axis and near 0 , there is a dense set of estimates indicated by many markers ' $x$ ' being very close together. Numerical estimates for 200 randomly perturbed versions of the matrix in (2) are also plotted (blue '.'). According to Lemma 1, exact eigenvalues are real. So complex eigenvalues here are wrong: numerical methods fail on this class of graph Laplacians. 
To explore the pseudospectra in a way that avoids the three violations of the underlying model, here we propose to perturb only a subset of the entries of the matrix (2), and in a way that respects the graph Laplacian structure and chemical structure. This perturbation is done by adding the absolute value of independent and identically distributed samples from the standard normal distribution to each positive entry of (2), and then adjusting the negative main diagonal entries to preserve the graph Laplacian property. Figure 1 illustrates an example of our proposal, but visually, this appears very similar to what one might obtain by the usual pseudospectra. A plausible explanation of that similarity is that the figure is still computed by standard eigenvalue algorithms, which can only return estimates that approximately correspond to the usual pseudospectra of the input matrix to the algorithm (and although the class of input matrices are structurally different to what is usually input, they are intuitively still 'close'). Random matrix theory has been applied to non-symmetric graph Laplacians by Timm [13], and specifically to Twisted Toeplitz matrices by Basak, Paquette, and Zeitouni [1].

Remark 3. Finding eigenvalues of a $4 \times 4$ version of the matrix (2) (corresponding to $\mathrm{m}=3$ and the case labelled $(3,3)$ in the experiments of Figure $4(\mathrm{f})$ of Shon and Cohen [12]), with symbolic software in Wolfram Alpha ${ }^{1}$ produces a complicated expression, involving imaginary units:

$$
-\frac{1 \pm i \sqrt{3}}{6^{3} \sqrt{2}}\left[4\left(-3 c_{2}^{2}+6 c_{1} c_{2}-49 c_{1}^{2}\right)^{3}+\cdots\right]^{1 / 2}+\cdots .
$$

Presumably this is an artefact of the Wolfram Alpha software using Cardano's formula for an associated cubic. If not aware of Lemma 1, then a naive user of symbolic algebra software might easily be misled.

From standard theory of Markov processes it is known that matrices such as (2) have a unique zero eigenvalue, and that all other eigenvalues have negative real part. Together with Lemma 1, we see that all nonzero eigenvalues are negative.

${ }^{1}$ https://www.wolframalpha.com/, accessed 20/02/2020 
After factoring out the zero eigenvalue, the characteristic polynomial is reduced in degree by one. Hence we find the eigenvalues of the $3 \times 3$ case with the quadratic formula, and the $4 \times 4$ case with Cardano's formula for the cubic. (And the $5 \times 5$ case via formulae for a quartic, but not $6 \times 6$ or larger cases, which lead to polynomials of higher degree.) Thus we now have a procedure that finds exact eigenvalues for the cases in all of the experiments in Figure 4(f) of Shon and Cohen [12].

Having found an eigenvalue, the corresponding right eigenvector can always be found by solving $\mathbb{A} v=\lambda v$ in a recursive way, analogous to the method of forward substitution used in Gaussian elimination, to take advantage of the tridiagonal structure. In particular, set $v_{1}=1$, then set $v_{2}=v_{1}\left(\lambda-a_{11}\right) / a_{12}$, and then recursively

$$
v_{i+1}=\left[-a_{i, i-1} v_{i-1}+\left(\lambda-a_{i i}\right) v_{i}\right] / a_{i, i+1} .
$$

Thus we have a procedure to exactly diagonalise the examples in all of the experiments in Figure 4(f) of Shon and Cohen [12], mentioned above.

The stationary distribution (the right eigenvector for $\lambda=0$ ) can always be found in the way described above, and this distribution is well known for this class of bimolecular models [11, Sec. 1.2, 1.6]. Even more generally than the class of models we study in this article, according to the Matrix-Tree Theorem [4], for all matrices of the graph Laplacian type there are known formulae for the vector in the null space.

An illustrative example for the $m=1$ case with $\lambda_{0}=0, \lambda_{1}=-\left(c_{1}+c_{2}\right)$ has an exact diagonalization

$$
\left(\begin{array}{cc}
-c_{1} & c_{2} \\
c_{1} & -c_{2}
\end{array}\right)=\frac{1}{\sqrt{\left|\lambda_{1}\right|}}\left(\begin{array}{cc}
c_{2} & 1 \\
c_{1} & -1
\end{array}\right)\left(\begin{array}{cc}
\lambda_{0} & 0 \\
0 & \lambda_{1}
\end{array}\right)\left(\begin{array}{cc}
1 & 1 \\
c_{1} & -c_{2}
\end{array}\right) \frac{1}{\sqrt{\left|\lambda_{1}\right|}} .
$$

In another example, for the $m=2$ case, let $\lambda_{0}, \lambda_{+}$and $\lambda_{-}$denote the three eigenvalues, and let $v_{0}, v_{+}$and $v_{-}$denote the corresponding eigenvectors. Exact eigenvalues and eigenvectors are

$$
\lambda_{0}=0 \text { and } v_{0}=\left(1,4 c_{1} / c_{2}, 2 c_{1}^{2} / c_{2}^{2}\right),
$$


and

$$
\begin{aligned}
& \lambda_{ \pm}=\frac{1}{2}\left(-5 c_{1}-3 c_{2} \pm \sqrt{\left(c_{1}-c_{2}\right)^{2}+8 c_{1}^{2}}\right) \\
& v_{ \pm}=\left(1,\left(4 c_{1}+\lambda_{ \pm}\right) / c_{2}, c_{1}\left(4 c_{1}+\lambda_{ \pm}\right) /\left[c_{2}\left(2 c_{2}+\lambda_{ \pm}\right)\right]\right) .
\end{aligned}
$$

The autocorrelation function in equation (5) of Shon and Cohen [12] is used to estimate the rate constants from experimental observations. That autocorrelation involves the matrix exponential of (1), and in the $2 \times 2$ case this is available as an algebraic expression, as noted above, but for larger matrices this matrix exponential is numerically estimated by Shon and Cohen [12]. The procedure for exact diagonalizations we describe above, for say the $3 \times 3$ case, allows expressions for the matrix exponential to be found without resorting to numerical calculation - allowing the dependence on parameters to be made explicit.

\section{Application of the Magnus expansion}

We now consider the case that the rate 'constants' $c_{1}=c_{1}(t)$ and $c_{2}=c_{2}(t)$ in (2) are in fact time dependent, to model experiments in which these rates vary. Then the matrix varies in time, $\mathbb{A}=\mathbb{A}(t)$. Instead of $(1)$, we now have

$$
\frac{d}{d t} p=\mathbb{A}(t) p \quad \text { with solution } \quad p(t)=\exp (\Omega(t)) p(0) .
$$

Here, $\Omega(t)$ is given by the Magnus expansion $[2,5]$ :

$$
\Omega(t)=\int_{0}^{t} \mathbb{A}(s) d s-\frac{1}{2} \int_{0}^{t}\left[\int_{0}^{s} \mathbb{A}(r) d r, \mathbb{A}(s)\right] d s+\cdots .
$$

Here the commutator $[X, Y]$ of two matrices $X$ and $Y$ is defined to be $[X, Y]=$ $X Y-Y X$. All higher order terms (not shown in (4)) involve commutators. For example, the next two terms of (4) are

$$
+\frac{1}{12} \int_{0}^{t}\left[\int_{0}^{s_{1}} \mathbb{A}\left(s_{2}\right) d s_{2},\left[\int_{0}^{s_{1}} \mathbb{A}\left(s_{2}\right) d s_{2}, \mathbb{A}\left(s_{1}\right)\right]\right] d s_{1}
$$


and

$$
+\frac{1}{4} \int_{0}^{t}\left[\int_{0}^{s_{1}}\left[\int_{0}^{s_{2}} \mathbb{A}\left(s_{3}\right) \mathrm{d} s_{3}, \mathbb{A}\left(s_{2}\right)\right] d s_{2}, \mathbb{A}\left(s_{1}\right)\right] d s_{1} .
$$

For a practical numerical method, only a few terms of the infinite Magnus series (4) can be retained $[2,5]$. For example, a second-order method is obtained by keeping only the first term of the series in (4), and by approximating the integral by the midpoint rule via $\int_{0}^{h} \mathbb{A}(t+s) d s \approx h \mathbb{A}(t+h / 2)$. Thus with

$$
\sigma=h \mathbb{A}(t+h / 2),
$$

we take a small time step $h>0$ in the second-order numerical scheme via

$$
\hat{p}(t+h)=\exp (\sigma) \hat{p}(t) .
$$

An attractive property of the second-order method in (5) is that it does not require matrix commutators [2,5], and that it respects the nonnegative property of solutions because $\sigma=h \mathbb{A}(t+h / 2)$ is a graph Laplacian. A fourth-order method is obtained by including just one commutator $[2,5]$ : with $\sigma=\frac{1}{2} h\left(A_{1}+A_{2}\right)+\frac{\sqrt{3}}{12} h^{2}\left[A_{2}, A_{1}\right]$, where $A_{1}=\mathbb{A}(t+(1 / 2-\sqrt{3} / 6) h)$ and $A_{2}=\mathbb{A}(t+(1 / 2+\sqrt{3} / 6) h)$ are evaluated at Gauss quadrature points, we take a time step $h$ in the fourth-order numerical scheme via

$$
\widehat{p}(t+h)=\exp (\sigma) \hat{p}(t) .
$$

For our chemical application, the commutators can be substantially simplified. We have $\mathbb{A}(t)=c_{1}(t) M+c_{2}(t) N$ where $M$ and $N$ are both bidiagonal and constant matrices. Hence

$$
\left[\mathbb{A}\left(t_{1}\right), \mathbb{A}\left(t_{2}\right)\right]=\left[c_{1}\left(t_{1}\right) c_{2}\left(t_{2}\right)-c_{1}\left(t_{2}\right) c_{2}\left(t_{1}\right)\right][M, N] .
$$

The point is that the commutator $\left[\mathbb{A}\left(t_{1}\right), \mathbb{A}\left(t_{2}\right)\right]$ required by the Magnus expansion and numerical scheme, involves constant matrices. Thus $[\mathrm{M}, \mathrm{N}]$ are precomputed once. All other computations only involve scalar functions $\mathbf{c}_{1}(\mathrm{t})$, 
$c_{2}(t)$, which represents a considerable numerical savings. MacNamara and Burrage [8] present an example of this application.

The advantage of including the commutator is the significant increase in the order of accuracy. For our particular class of problems, a disadvantage is that usually, a commutator of two graph Laplacian matrices is not a graph Laplacian. Notice that in (7), up to the scalar factor $\left[\mathbf{c}_{1}\left(\mathbf{t}_{1}\right) \mathbf{c}_{2}\left(\mathbf{t}_{2}\right)-\right.$ $\left.c_{1}\left(t_{2}\right) c_{2}\left(t_{1}\right)\right]$, the commutator $\left[\mathbb{A}\left(t_{1}\right), \mathbb{A}\left(t_{2}\right)\right]$ is proportional to $\pm[M, N]$. For example, in the $3 \times 3$ case $(\mathrm{m}=2)$ we have

$$
[M, N]=\left[\begin{array}{ccc}
-4 & -3 & 0 \\
4 & 2 & -2 \\
0 & 1 & 2
\end{array}\right]
$$

This evidently does not exhibit the pattern of signs of a graph Laplacian! Hence $\left[\mathbb{A}\left(t_{1}\right), \mathbb{A}\left(t_{2}\right)\right]$ in $(7)$ is not a graph Laplacian. This matters because graph Laplacian matrices $L$ have the special property that all entries of the matrix exponential $\exp (\mathrm{L})$ are nonnegative and columns are probability vectors, which is important to the probabilistic interpretation of the model. Unfortunately, the commutator does not have the same pattern of signs as the matrix $\mathbb{A}$, and the commutator is not a graph Laplacian, so although (6) is fourth-order, the numerical method (6) could produce negative numbers (while the true solution is positive).

Fourth-order commutator-free quasi-Magnus integrator To avoid commutators that are not graph Laplacian matrices appearing in the exponent of the Magnus integrators, we now consider commutator-free quasi-Magnus integrators. For example, consider the averaged matrices

$$
\sigma_{1}=\frac{1}{2} h\left(\alpha A_{1}+\beta A_{2}\right), \quad \sigma_{2}=\frac{1}{2} h\left(\beta A_{1}+\alpha A_{2}\right),
$$

with

$$
A_{1}=\mathbb{A}(t+(1 / 2-\sqrt{3} / 6) h), \quad A_{2}=\mathbb{A}(t+(1 / 2+\sqrt{3} / 6) h)
$$


and

$$
\alpha=\frac{1}{2}+\frac{\sqrt{3}}{3}, \quad \beta=\frac{1}{2}-\frac{\sqrt{3}}{3},
$$

for a small time step $h>0$. Note that $\alpha+\beta=1, \beta<0$ and $\alpha /|\beta|=$ $7+4 \sqrt{3} \simeq 14$. The point is that $\beta$ is negative, but much smaller than $\alpha$. Then, unless $\mathbb{A}(t)$ changes with $t$ suddenly, this scheme has the attractive property that $\sigma_{1}$ and $\sigma_{2}$ will remain graph Laplacian matrices. To give a sense that most practical examples will not change too suddenly, we now give an example of how to be certain that $\sigma_{1}$ and $\sigma_{2}$ will indeed remain graph Laplacian matrices.

A fourth-order integrator in the time step $h$ is given by the numerical scheme [2, and references therein]

$$
\hat{p}(t+h)=\exp \left(\sigma_{2}\right) \exp \left(\sigma_{1}\right) \hat{p}(t) .
$$

Each exponential is interpreted as a scheme to advance a half step with an averaged method followed by the averaged adjoint method, such that the result is accurate up to order four. To illustrate the application of our recommended numerical method (8) with our running example, let us suppose that $c_{1}=c_{1}(t)$ and $c_{2}$ is constant. The only constraint for $\sigma_{1}, \sigma_{2}$ to be graph Laplacian matrices is that

$$
\max \left\{\frac{c_{1}\left(t_{1}\right)}{c_{1}\left(t_{2}\right)}, \frac{c_{1}\left(t_{2}\right)}{c_{1}\left(t_{1}\right)}\right\}<7+4 \sqrt{3} \simeq 14 .
$$

If $c_{1}(t)$ is a smooth function, then this is a condition that should be satisfied for all time steps of practical interest. Moreover, this condition is always guaranteed with simple adaptive time stepping.

\section{Conclusions}

We have applied techniques for graph Laplacians to a particular application that is motivated by single molecule technology and experiments with the 
Dimple Machine [12]. We have given reasons why the usual way of studying the pseudospectra has a drawback for graph Laplacians, and therefore we have proposed a different way to interrogate (an approximation to) the pseudospectra, which we illustrate with the Monte Carlo dot cloud of Figure 1.

We explained that exact diagonalizations are possible via the procedure we exemplified for some of the experiments, which can give exact solutions. However, the exact solution to the bimolecular master equation with $m=5$ or more molecules of each species $S_{1}, S_{2}$, and assocciated eigenvalues of (2) remains an important open question.

Finally, we suggest how the Magnus expansion can be applied to simulations of bimolecular reactions when rates vary in time. The recurring theme of the article is the graph Laplacian structure, and again this issue manifests itself in the commutators arising in the infinite expansion (4). Standard numerical methods are not able to respect key properties of the solution, such as nonnegativity. We address this issue by showing how a fourth-order method can be achieved while simultaneously maintaining positivity. Preserving graph Laplacian structures and associated properties in numerical schemes is a much wider issue than only the application we focused on here, and an important future challenge.

Acknowledgements We thank the organisers and delegates of the Canberra 2019 EMAC conference for helpful discussions about graph Laplacians. SM thanks the Australian Research Council Centre of Excellence for Mathematical and Statistical Frontiers (ACEMS). The work of SB was funded by Ministerio de Economía, Industria y Competitividad (Spain) through project MTM2016-77660-P (AEI/FEDER, UE).

\section{References}

[1] A. Basak, E. Paquette, and O. Zeitouni. "Regularization of non-normal matrices By Gaussian noise - The banded Toeplitz And twisted 
Toeplitz cases". In: Forum of Mathematics, Sigma. Vol. 7. Cambridge University Press. 2019. DOI: doi:10.1017/fms.2018.29 (cit. on p. C65).

[2] S. Blanes, F. Casas, J. A. Oteo, and J. Ros. "The Magnus expansion and some of its applications". In: Phys. Rep. 470.5-6 (2009), pp. 151-238. DOI: 10.1016/j . physrep.2008.11.001 (cit. on pp. C67, C68, C70).

[3] B. A. Earnshaw and J. P. Keener. "Invariant manifolds of binomial-like nonautonomous master equations". In: SIAM J. Appl. Dyn. Sys. 9.2 (2010), pp. 568-588. DOI: 10.1137/090759689 (cit. on p. C61).

[4] J. Gunawardena. "A linear framework for time-scale separation in nonlinear biochemical systems". In: PloS One 7.5 (2012), e36321. DOI: 10.1371/journal. pone.0036321 (cit. on p. C66).

[5] A. Iserles and S. MacNamara. "Applications of Magnus expansions and pseudospectra to Markov processes". In: Euro. J. Appl. Math. 30.2 (2019), pp. 400-425. DOI: 10.1017/S0956792518000177 (cit. on pp. C61, C67, C68).

[6] S. MacNamara. "Cauchy integrals for computational solutions of master equations". In: ANZIAM Journal 56 (2015), pp. 32-51. DOI: 10.21914/anziamj.v56i0.9345 (cit. on p. C63).

[7] S. MacNamara, A. M. Bersani, K. Burrage, and R. B. Sidje. "Stochastic chemical kinetics and the total quasi-steady-state assumption: Application to the stochastic simulation algorithm and chemical master equation". In: J. Chem. Phys. 129 (2008), p. 095105. DOI: 10.1063/1.2971036 (cit. on p. C63).

[8] S. MacNamara and K. Burrage. "Stochastic modeling of naive T cell homeostasis for competing clonotypes via the master equation". In: SIAM Multiscale Model. Sim. 8.4 (2010), pp. 1325-1347. DOI: 10.1137/09077182X (cit. on p. C69). 
[9] S. MacNamara, K. Burrage, and R. B. Sidje. "Multiscale modeling of chemical kinetics via the master equation". In: SIAM Multiscale Model. ES Sim. 6.4 (2008), pp. 1146-1168. DOI: 10.1137/060678154 (cit. on p. C63).

[10] S. MacNamara, Wi. McLean, and K. Burrage. "Wider contours and adaptive contours". In: 2017 MATRIX Annals. Ed. by J. de Gier, C. E. Praeger, and T. Tao. Springer International Publishing, 2019, pp. 79-98. DOI: 10.1007/978-3-030-04161-8_7 (cit. on p. C63).

[11] M. J. Shon. "Trapping and manipulating single molecules of DNA". PhD thesis. Harvard University, 2014. URL:

http://nrs . harvard.edu/urn-3:HUL. InstRepos:11744428 (cit. on pp. C60, C66).

[12] M. J. Shon and A. E. Cohen. "Mass action at the single-molecule level". In: J. Am. Chem. Soc. 134.35 (2012), pp. 14618-14623. DOI: 10.1021/ja3062425 (cit. on pp. C60, C61, C62, C65, C66, C67, C71).

[13] C. Timm. "Random transition-rate matrices for the master equation". In: Phys. Rev. E 80.2 (2009), p. 021140. DOI: 10.1103/PhysRevE.80.021140 (cit. on p. C65).

[14] L. N. Trefethen and M. Embree. Spectra and pseudospectra: The behavior of nonnormal matrices and operators. Princeton University Press, 2005. URL: https://press.princeton. edu/books/ hardcover/9780691119465/spectra-and-pseudospectra (cit. on pp. C62, C63).

\section{Author addresses}

1. S. MacNamara, Australian Research Council Centre of Excellence for Mathematical and Statistical Frontiers (ACEMS), School of Mathematical and Physical Sciences, University of Technology Sydney, NSW 2007, Australia.

mailto:shev.macnamara@uts.edu.au 
2. Sergio Blanes, Instituto de Matemática Multidisciplinar, Universitat Politècnica de València, Edificio 8-G, piso 2, Camino de Vera s/n, 46022-Valencia mailto:serblaza@imm.upv.es

3. Arieh Iserles, Department of Applied Mathematics and Mathematical Physics, University of Cambridge, Wilberforce Road, Cambridge CB3 0WA, UK mailto:a.iserles@damtp.cam.ac.uk 